\title{
Treatment of advanced stage cholangiocarcinoma: Systemic therapy may be the starting step for radical surgery
}

\author{
HyungJoo Baik', Sang Hyuk Seo ${ }^{1}$, Kwang Hee Kim ${ }^{1}$, Moon Young Choi ${ }^{2}$, Yo-Han Park', Chang Soo Choi ${ }^{1}$ \\ Departments of ${ }^{1}$ Surgery and ${ }^{2}$ Internal Medicine, Inje University Busan Paik Hospital, Inje University College of Medicine, Busan, Korea
}

\begin{abstract}
Cholangiocarcinoma is the most common biliary malignancy and the second most common hepatic malignancy following hepatocellular carcinoma. It can be classified anatomically as intrahepatic cholangiocarcinoma (iCCA), perihilar CCA, or distal CCA. The treatment of choice for iCCA is surgical resection, but only those with potentially resectable tumors can undergo surgery. The standard regimen for advanced stage iCCA is gemcitabine and cisplatin. We encountered two unusual cases of iCCA patients who were initially diagnosed as unresectable cases and underwent systemic chemotherapy, which showed great response and therefore enabled radical operation. The patients show that even though iCCA is a challenging disease, patients with good response to chemotherapy may have a chance to undergo radical surgery.
\end{abstract}

Keywords: Cholangiocarcinoma, Gemcitabine, Cisplatin, Cholangiocarcinoma, Chemotherapy

\section{INTRODUCTION}

Cholangiocarcinoma is the most common biliary malignancy and the second most common hepatic malignancy following hepatocellular carcinoma. The treatment of choice for intrahepatic cholangiocarcinoma (iCCA) is surgical resection; however, many patients are diagnosed at unresectable state. For advanced stage patients, the standard regimen is gemcitabine and cisplatin, proven to be efficient by the ABC- 02 trial [1,2]. We encountered two unusual cases of iCCA patients who were initially diagnosed as advanced stage and underwent systemic chemotherapy, which enabled radical operation.

Received: Apr 4, 2018 Accepted: Jun 6, 2018

Correspondence to: Yo-Han Park

Division of Hepato-Pancreato-Biliary Surgery, Department of Surgery, Inje University Busan Paik Hospital, Inje University College of Medicine, 75

Bokji-ro, Busanjin-gu, Busan 47392, Korea

Tel: +82-51-890-6352, Fax: +82-51-898-9427

E-mail: prientempt@naver.com

Copyright @ C Korean Society of Surgical Oncology

This is an Open Access article distributed under the terms of the Creative Commons Attribution Non-Commercial License (http://creativecommons.org/licenses/by-nc/4.0) which permits unrestricted non-commercial use, distribution, and reproduction in any medium, provided the original work is properly cited.

\section{CASE REPORTS}

\section{Case 1}

Patient A is a 68-year-old female with history of common bile duct stone in 2005, which was removed by endoscopic retrograde cholangiopancreatogram (ERCP). The patient presented with fever, chilling, and right upper quadrant abdominal pain that had lasted 1 week. Initial laboratory findings were as follows: total bilirubin, $0.5 \mathrm{mg} / \mathrm{dL}$; aspartate transaminase/alanine transaminase (AST/ ALT), 107/141 IU/L; alkaline phosphatase/gamma-glutamyl transpeptidase (ALP/ $\gamma$-GTP), 725/168 U/L; CA19-9, 38.07 U/mL. Biliary computed tomography (CT) showed $3.6 \mathrm{~cm}$ cholangiocellular carcinoma at left lobe with underlying chronic cholangitis. Positron emission tomography (PET)/CT showed diffuse 18F-fluorodeoxyglucose (FDG) uptake in left lobe and S1, with possible ingrowth into right lobe, as well as possible metastatic lymphadenopathy in anterior diaphragmatic area (Fig. 1D). Intrahepatic duct biopsy using ERCP confirmed poorly differentiated adenocarcinoma. Because of PET/CT findings, the patient received palliative chemotherapy using gemcitabine-cisplatin. After the third cycle, the size of main mass had decreased from $3.6 \mathrm{~cm}$ to $2.2 \mathrm{~cm}$; after the sixth, the tumor had become as small as $1.6 \mathrm{~cm}$ (Fig. 1), and CA19-9 has decreased to $1.44 \mathrm{U} / \mathrm{mL}$ after chemotherapy. On 

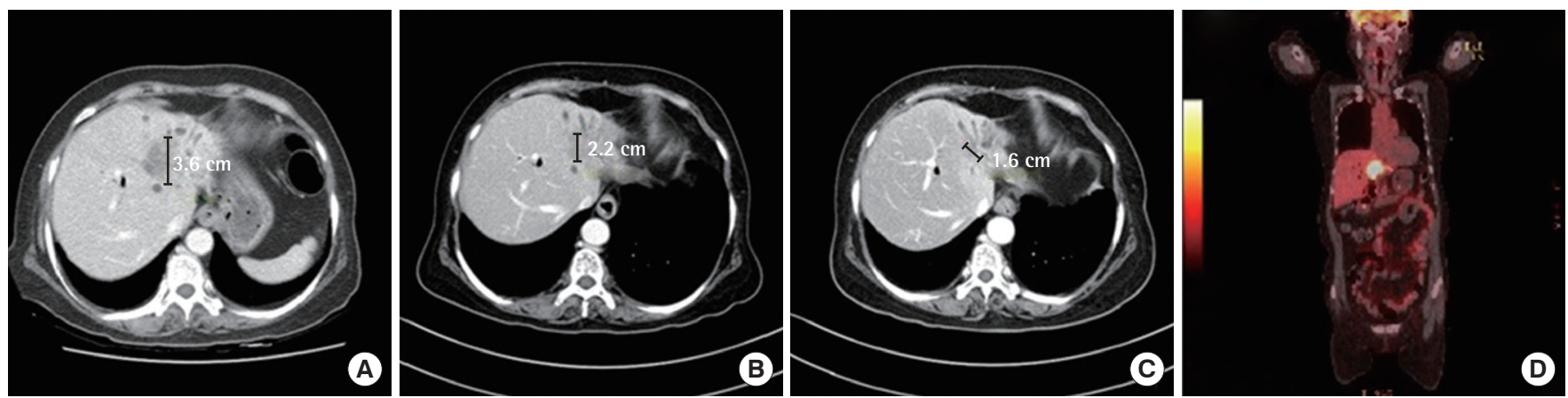

Fig. 1. Effect of gemcitabine-cisplatin chemotherapy on patient A. (A) Initial tumor size was $3.6 \mathrm{~cm}$; (B) after three cycles of chemotherapy, the tumor had decreased to $2.2 \mathrm{~cm}$; (C) after six cycles of chemotherapy, the tumor had become as small as $1.6 \mathrm{~cm}$. (D) Patient A with intrahepatic cholangiocarcinoma at left lobe and S1, with possible ingrowth into right lobe, and possible metastatic lymphadenopathy in anterior diaphragmatic area on positron emission tomography/computed tomography.

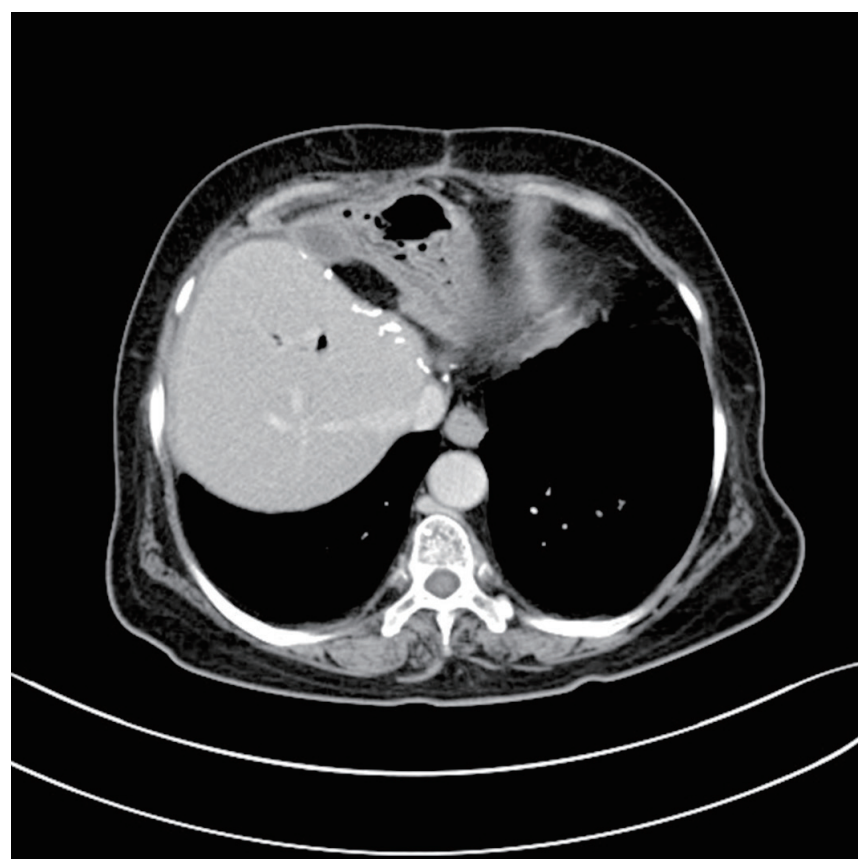

Fig. 2. Postoperative computed tomography of patient $A$ after left lobectomy with $\mathrm{S} 1$ resection.

May 2016, the patient received left lobectomy with S1 resection. Grossly there was no tumor like lesion on surface, but after dissection, there were mass like lesion nearby hilar area. Final pathology reported of no residual carcinoma, with marked inflammatory reaction and fibrosis in the portal areas. After the 10th cycle of chemotherapy, the patient had grade 3 anemia and grade 2 peripheral neuropathy, so cisplatin was omitted thereafter. The patient continued with her chemotherapy up to the 13th cycle without recurrence, so she has stopped chemotherapy and is going through close follow-up without recurrence, 16 months postoperatively (Fig. 2).

\section{Case 2}

Patient B is a 69-year-old female with past history of intrahepatic bile duct stone, with possibility of choledochoduodenostomy 30 years ago. She presented with right upper quadrant abdominal pain that had lasted for more than 2 months. Initial laboratory findings were as follows: total bilirubin, $0.6 \mathrm{mg} / \mathrm{dL}$; AST/ALT, 65/37 IU/L; ALP/ $\gamma$-GTP, 717/140 U/L; CA19-9, 570 U/mL. Biliary CT showed pyogenic cholangitis with $3.1 \mathrm{~cm}$ mass at liver left lateral segment along with $1.3 \mathrm{~cm}$ enhancing nodule at gastrohepatic area and $1.5 \mathrm{~cm}$ rim-enhancing nodule at anterior aspect of left lobe, indicating iCCA with extrahepatic metastasis. Sono-guided liver biopsy confirmed well-differentiated adenocarcinoma. PET/CT suggested right cardiophrenic metastasis (Fig. 3D); thus, the patient underwent gemcitabine-cisplatin chemotherapy. After the third cycle, the tumor had decreased in size from $3.1 \mathrm{~cm}$ to $2.8 \mathrm{~cm}$; after the sixth cycle, the tumor had become $2.5 \mathrm{~cm}$ (Fig. 3), and CA19-9 has decreased to $157.1 \mathrm{U} / \mathrm{mL}$ after chemotherapy. PET/CT also suggested partial response in both the main mass and the metastatic nodules. On December 2016, the patient received left lateral sectionectomy with lymphadenectomy at areas 1 and 8 . The area where cardiophrenic invasion was suspected was shaved off for maximum removal of possible metastasis. Final pathology reported of classical adenocarcinoma, $2.5 \mathrm{~cm}$ in size with clear margins, serosal, lymphatic, and perineural invasion. Four out of 11 lymph nodes had metastasis. The patient had her eighth gemcitabine-cisplatin and is still undergoing close follow-up without evidence of recurrence, 9 months postoperatively (Fig. 4).

\section{DISCUSSION}

Cholangiocarcinomas are rare but one of the most difficult carcinomas to treat. Most cases of cholangiocarcinoma patients are di- 

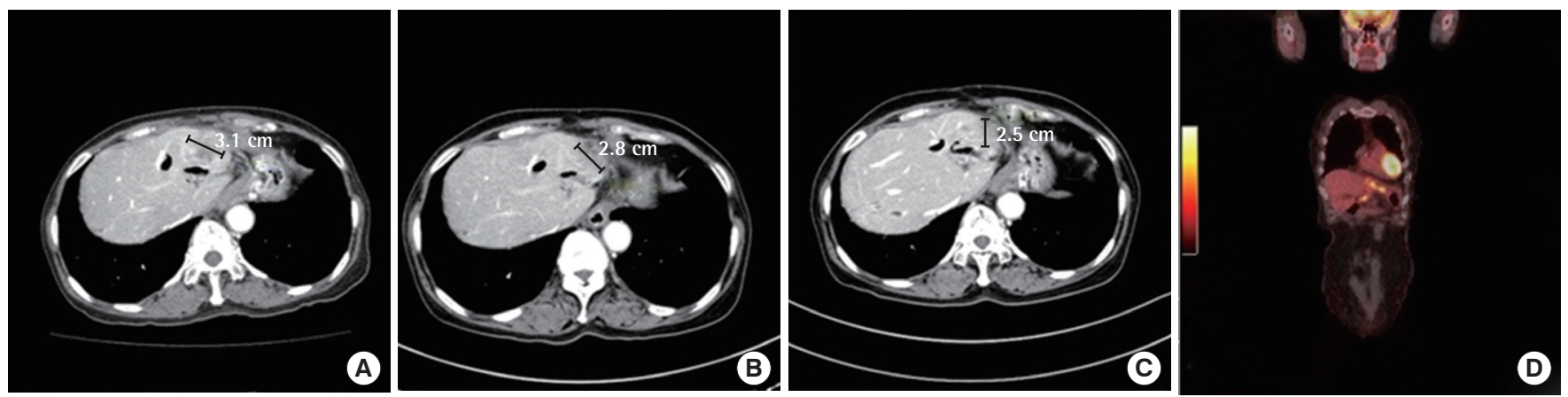

Fig. 3. Effect of gemcitabine-cisplatin chemotherapy on patient B. (A) The patient had her tumor on left lateral segment of liver, sized 3.1 $\mathrm{cm}$, as well as an enhancing nodule at gastrohepatic area. (B) After three cycles of chemotherapy the tumor had decreased to $2.8 \mathrm{~cm}$; (C) after six cycles of chemotherapy, both the tumor and gastrohepatic lymph node metastasis had decreased. (D) Patient B with intrahepatic cholangiocarcinoma at left lobe with right cardiophrenic metastasis on positron emission tomography/computed tomography.

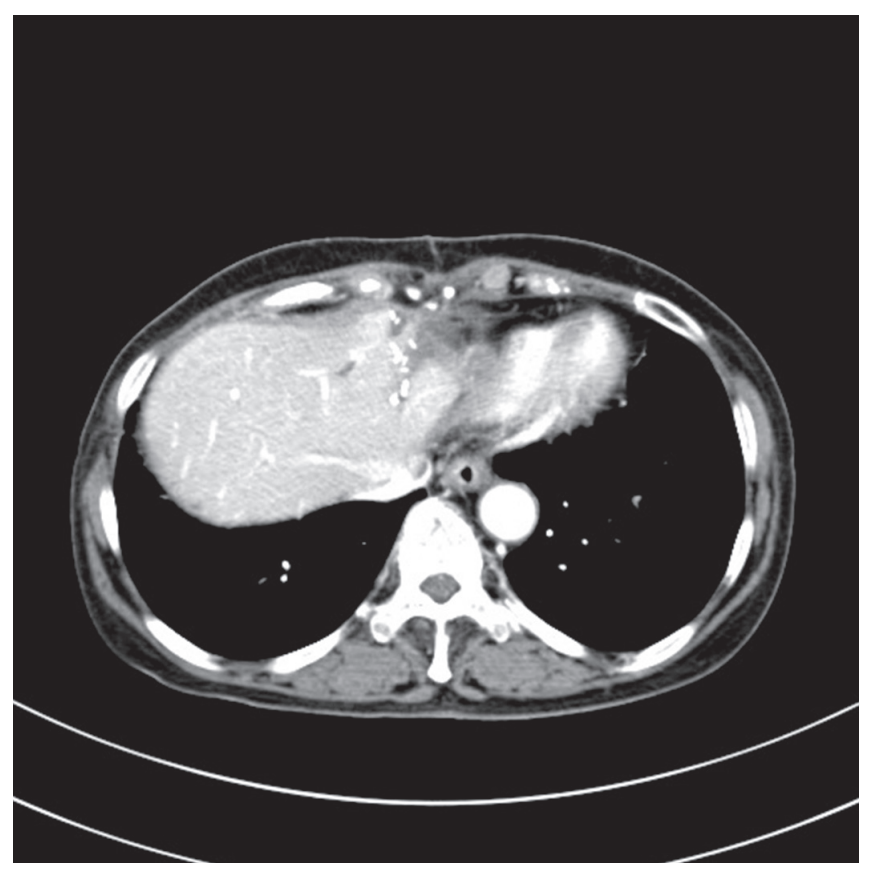

Fig. 4. Postoperative computed tomography of patient B after left lateral sectionectomy with lymphadenectomy.

agnosed with either imaging studies or symptoms such as jaundice, which present at an advanced stage. The incidence of iCCA is increasing, accounting for $10 \%$ of all biliary cancers, whereas that of extrahepatic CCA (eCCA) is decreasing, suggesting that different risk factors may be involved. In fact, there is evidence that the protein and receptor expression of eCCA and iCCA may differ due to different carcinogenic pathways [3]. Due to its favor for vascular invasion and rapid progression, iCCA is associated with poor prognosis. Surgical management of iCCA involves hepatectomy and adequate lymph node dissection in effort to achieve negative microscopic margins while preserving adequate remnant liver vol- ume. Negative margin is the most important variable associated with outcome [4]. It has been stated in textbook that neither chemotherapy nor radiation therapy has shown to improve survival in advanced cholangiocarcinomas [5]. However, recent studies have shown positive data regarding palliative chemotherapy. In Grendar's review article [6], the authors conclude that there is no evidence to suggest improved outcomes with neoadjuvant chemotherapy in resectable patients; however, in primarily unresectable patients, there is evidence to suggest improve R0 resectability after neoadjuvant therapy and improved survival when followed by surgery. In the ABC-02 trial, patients with gemcitabine-cisplatin chemotherapy had longer median overall survival, progression-free survival, and rate of tumor control than patients treated with gemcitabine alone. The median survival in the gemcitabine-cisplatin group was 11.7 months compared to that of gemcitabine-only group, with 8.1 months $(\mathrm{P}<0.001)$; the median progression-free survival was 8.0 months in gemcitabine-cisplatin compared with 5.0 months in the gemcitabine-only group $(\mathrm{P}<0.001)$ [1]. Okusaka et al. [7] have also shown a superior benefit with the gemcitabine-cisplatin combination regimen compared with gemcitabine-only group in terms of disease control rate, overall survival, and progression-free survival. Some Japanese surgeons have also reported of advanced iCCA patients who received neoadjuvant chemotherapy, then had the tumors surgically removed $[8,9]$. Furthermore, complete pathologic response to neoadjuvant chemotherapy in cholangiocarcinoma is extremely rare, with only two case reports in the English language for iCCA [10,11]. Our two patients, especially patient A whose pathologic report showed complete pathologic response, have shown significant reduction in tumor volume by neoadjuvant chemotherapy which have made curative resection possible. These cases, along with ours, suggest that advanced biliary tract cancers may have some response to 
chemotherapy; therefore, aggressive treatment should not be neglected. Target-oriented agents in combination with gemcitabine based regimen are actively under studies, but so far there are no or only marginal benefits from numerous trials [12]. Larger studies with chemotherapy and target agents are necessary to validate as the mainstay of treatment in biliary tract cancers.

In conclusion, cholangiocarcinoma is an aggressive malignancy that has limited treatment options and low survival rates. However, if patients are responsive to chemotherapy, surgical management should be followed for radical excision of tumor, as it may improve overall survival.

\section{CONFLICT OF INTEREST}

No potential conflict of interest relevant to this article was reported.

\section{REFERENCES}

1. Valle J, Wasan H, Palmer DH, Cunningham D, Anthoney A, Maraveyas A, et al. Cisplatin plus gemcitabine versus gemcitabine for biliary tract cancer. N Engl J Med 2010;362:1273-81.

2. Bridgewater J, Lopes A, Palmer D, Cunningham D, Anthoney A, Maraveyas A, et al. Quality of life, long-term survivors and longterm outcome from the ABC-02 study. Br J Cancer 2016;114:96571.

3. Aljiffry M, Walsh MJ, Molinari M. Advances in diagnosis, treatment and palliation of cholangiocarcinoma: 1990-2009. World J Gastroenterol 2009;15:4240-62.

4. DeOliveira ML, Cunningham SC, Cameron JL, Kamangar F, Winter JM, Lillemoe KD, et al. Cholangiocarcinoma: thirty-one-year experience with 564 patients at a single institution. Ann Surg 2007; 245:755-62
5. Townsend CM Jr., Beauchamp RD, Evers BM, Mattox K, editors. Sabiston textbook of surgery: the biological basis of modern surgical practice. 20th ed. Philadelphia, PA: Elsevier Saunders; 2016.

6. Grendar J, Grendarova P, Sinha R, Dixon E. Neoadjuvant therapy for downstaging of locally advanced hilar cholangiocarcinoma: a systematic review. HPB (Oxford) 2014;16:297-303.

7. Okusaka T, Nakachi K, Fukutomi A, Mizuno N, Ohkawa S, Funakoshi A, et al. Gemcitabine alone or in combination with cisplatin in patients with biliary tract cancer: a comparative multicentre study in Japan. Br J Cancer 2010;103:469-74.

8. Otani T, Sakata J, Kameyama H, Otani A, Hirose Y, Tamura H, et al. Surgical resection after gemcitabine plus cisplatin chemotherapy for intrahepatic cholangiocarcinoma with multiple lymph node metastases: report of a case. Gan To Kagaku Ryoho 2016;43:17646.

9. Kamo N, Mori A, Nitta T, Hatano E, Mitsuyoshi H, Ikeda K, et al. Two cases of curatively resected intrahepatic cholangiocellular carcinomas through effective response to neoadjuvant chemotherapy. Gan To Kagaku Ryoho 2011;38:305-8.

10. Slupski MW, Szczylik C, Jasinski MK. Unexpected response to systemic chemotherapy in case of primarily nonresectable advanced disseminated intrahepatic cholangiocarcinoma. World J Surg On$\operatorname{col} 2007 ; 5: 36$

11. Tran TB, Bal CK, Schaberg K, Longacre TA, Chatrath BS, Poultsides GA. Locally advanced intrahepatic cholangiocarcinoma: complete pathologic response to neoadjuvant chemotherapy followed by left hepatic trisectionectomy and caudate lobectomy. Dig Dis Sci 2015;60:3226-9.

12. Sahu S, Sun W. Targeted therapy in biliary tract cancers-current limitations and potentials in the future. J Gastrointest Oncol 2017; 8:324-36 